\section{Laser Light Scattering Characterization of a Novel Polymer Nanofiber}

\author{
Chi Wu,*,t, Mei Li, ${ }^{\dagger}$ Simon Chi Man Kwan, ${ }^{\dagger}$ and \\ Guo J un Lius
}

Department of Chemistry, The Chinese University of Hong Kong, Shatin, Hong Kong, The Open Laboratory for Bond-Selective Chemistry, Department of Chemical Physics, University of the Science and Technology of China,

Hefei, Anhui, China, and Department of Chemistry,

The University of Calgary, Alberta, Canada, T2N 1N4

Recei ved J anuary 20, 1998

Revised Manuscript Received April 30, 1998

\section{Introduction}

It is well-known that a diblock copolymer may form a microphase separation in the bulk state due to the self-assembling of each block. Depending on the segment ratio of each block, the morphology could be spherical, cylindrical or lamellar. ${ }^{1}$ When dissolved in a selective solvent, which is a good solvent for one block, but poor for the other, diblock copolymers may form a core-shell micelle with the insoluble block as the core and the soluble block as the shell. ${ }^{2}$ If a solid substrate is in contact with this polymer solution, diblock copolymers may deposit on it to form a polymer brush when the solid/insoluble block interaction is favorable. ${ }^{3} \mathrm{Re}$ cently, Liu et al. ${ }^{4}$ prepared a novel type of nanofiber by photocrosslinking the PCEMA blocks in the self-assembled cylindrical phase of a polystyrene and polystyrene-b-poly(2-cinnamoylethyl methacrylate) (PS-b-PCEMA) diblock copolymer blend. The TEM graph showed that the nanofibers are $\sim 25 \mathrm{~nm}$ in diameter and $\sim 10^{3}$ $\mathrm{nm}$ in length. In this study, a combination of static and dynamic laser light scattering (LLS) was used to determine the mass and composition of such novel nanofibers as well as their conformation in solution.

\section{Experimental Section}

Sample Preparation. The polystyrene and poly(2-cinnamoylethyl methacrylate) blocks in the diblock copolymer, respectively, have 125 and 158 repeating units. The details of the copolymer synthesis and the nanofiber preparation were reported before. 4,5 Here, we only outline the nanofiber formation. A 1-mm thick copolymer film was prepared, in which PS-b-PCEMA formed a cylindrical phase. The film was irradiated half an hour on each side to cross-link the PCEMA blocks in the center of the cylindrical phase. The dissolution of polystyrene matrix in tetrahydrofuran (THF) led to a nanofiber suspension which contains soluble and insoluble portions. The TEM graph showed that both portions consisted of the nanofibers. In the insoluble portion, the nanofibers were longer and more entangled. In this study, only the soluble portion was investigated. The nanofiber concentration used was so dilute $\left(10^{-5}-10^{-6} \mathrm{~g} / \mathrm{mL}\right)$ that the overlapping of the nanofibers in the solution was avoided. The solutions were clarified by a $1 \mu \mathrm{m}$ pore size PTFE filter to remove dust before LLS measurements. There was no obvious flagging of the filter during the clarification and no obvious concentration change after the clarification.

Laser Light Scattering. A modified commercial LLS spectrometer (ALV/SP-125) equipped with an ALV-5000 multi- $\tau$

† The Chinese University of Hong Kong.

₹ University of the Science and Technology of China.

$\S$ The University of Calgary.

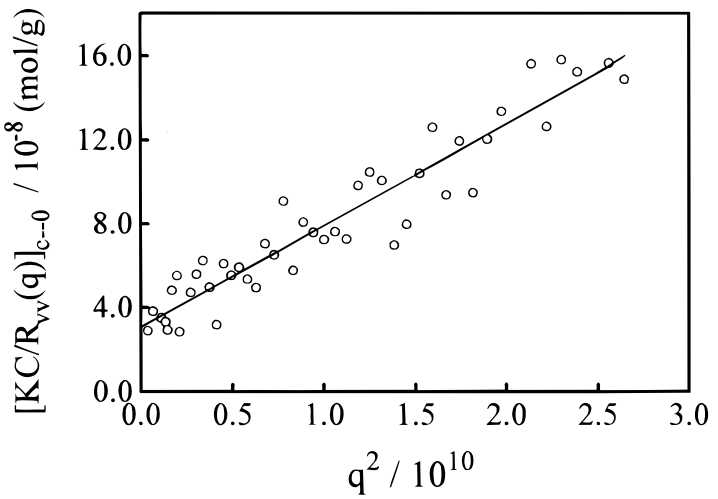

Figure 1. Angular dependence of the excess Rayleigh ratio $K C / R_{v v}(\theta)$ of the nanofibers in infinite dilution.

digital time correlator and a solid-state laser (ADLAS DPY $425 \mathrm{II}$, output power $\approx 400 \mathrm{~mW}$ at $\lambda_{0}=532 \mathrm{~nm}$ ) as the light source was used. The primary beam is vertically polarized with respect to the scattering plane. The instrument was calibrated with toluene to make sure that the scattering intensity has no angular dependence in the angular range $6-154^{\circ}$. The small angle range $\left(6-21^{\circ}\right)$ is particularly useful in this study because the nanofibers are quitelong. The detail of the LLS instrumentation and theory can be found else where. ${ }^{6,7}$ All the LLS measurements were done in toluene at $25.0 \pm 0.1^{\circ} \mathrm{C}$.

In static LLS, the angular dependence of the excess absolute time-averaged scattered intensity $R_{v v}(q)$, known as the excess Rayleigh ratio, was measured. For a dilute polymer solution measured at a low scattering angle, $R_{v v}(q)$ can be related to the weight-average molar mass $M_{w}$, the second virial coefficient $A_{2}$, and root-mean-square z-average radius of gyration $\left\langle\mathrm{R}_{\mathrm{g}}{ }^{2}\right\rangle_{\mathrm{z}}{ }^{1 / 2}$ (or written as $\left\langle\mathrm{R}_{\mathrm{g}}\right\rangle$ ) by ${ }^{8}$

$$
\frac{\mathrm{KC}}{\mathrm{R}_{\mathrm{vv}}(\mathrm{q})} \approx \frac{1}{\mathrm{M}_{\mathrm{w}}}\left(1+\frac{1}{3}\left\langle\mathrm{R}_{\mathrm{g}}{ }^{2}\right\rangle_{\mathrm{z}} \mathrm{q}^{2}\right)+2 \mathrm{~A}_{2} \mathrm{C}
$$

where $K=4 \pi^{2} n^{2}(d n / d C)^{2} /\left(N_{A} \lambda_{0}{ }^{4}\right)$ and $q=\left(4 \pi n / \lambda_{0}\right) \sin (\theta / 2)$ with $\mathrm{N}_{\mathrm{A}}, \mathrm{dn} / \mathrm{dC}, \mathrm{n}$, and $\lambda_{\mathrm{o}}$ being Avogadro's number, the specific refractive index increment, the solvent refractive index, and the wavelength of light in a vacuum, respectively. The value of $\mathrm{dn} / \mathrm{dC}$ was $0.11 \mathrm{~mL} / \mathrm{g} .^{5}$ In dynamic LLS, a precise intensityintensity time correlation function $\mathrm{G}^{(2)}(\mathrm{t}, \mathrm{q})$ in the self-beating mode was measured. The Laplace inversion of $\mathrm{G}^{(2)}(\mathrm{t}, \mathrm{q})$ can lead to a line width distribution $(G(\Gamma)){ }^{6}$

\section{Results and Discussion}

Figure 1 shows the angular dependence of the excess Rayleigh ratio of the nanofibers in infinite dilution. The values of $M_{w}, R_{g}$ and $A_{2}$ calculated on the basis of eq 1 are $3.3 \times 10^{7} \mathrm{~g} / \mathrm{mol}, 2200 \mathrm{~nm}$, and $\sim 7 \times 10^{-4} \mathrm{~mol} \cdot \mathrm{cm}^{3} /$ $\mathrm{g}^{2}$, respectively. Considering that individual diblock copolymer chains have a molar mass of $\sim 1.5 \times 10^{5}$ $\mathrm{g} / \mathrm{mol}$, we were able to estimate that each nanofiber consists of $\sim 220$ diblock copolymer chains. Our results showed that the depolarization ratio $\left(\rho \mathrm{v}=\left(\mathrm{R}_{\mathrm{vh}}(\theta) / \mathrm{R}_{\mathrm{v} v^{-}}\right.\right.$ $(\theta))$ in dilute solution was quite small, less than $5 \times$ $10^{-3}$, where $\mathrm{R}_{\mathrm{vh}}(\theta)$ and $\mathrm{R}_{\mathrm{vv}}(\theta)$ are the excess Rayleigh ratios measured by respectively putting a horizontal and a vertical polarizer in front of the detector. The ratio is related to the chain anisotropy $\delta$ by $\rho \mathrm{v}=3 \delta^{2} /(5+$ $\left.4 \delta^{2}\right)$. Nagai stated that all the measured LLS parameters have to be corrected; 9 namely, $\mathrm{M}_{\mathrm{w}}=\mathrm{M}_{\mathrm{w} \text {, mead }} / \gamma$,

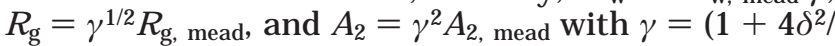




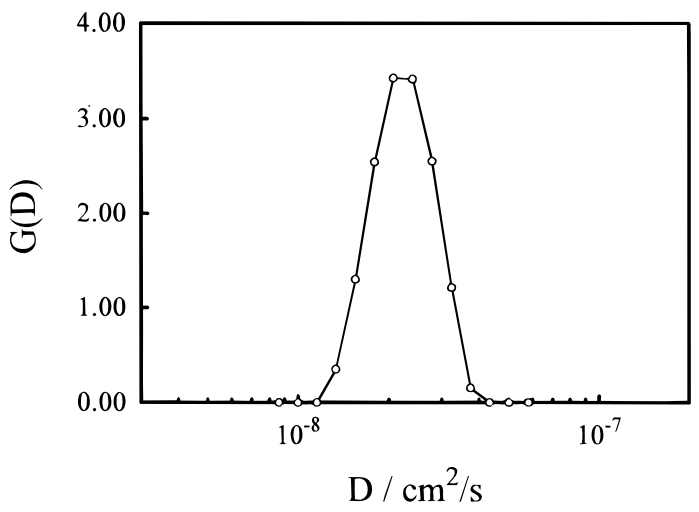

Figure 2. Typical translational diffusion coefficient distribution of the nanofiber in toluene, where the nanofiber concentration is $6 \times 10^{-6} \mathrm{~g} / \mathrm{mL}$ and the scattering angle is $6^{\circ}$.

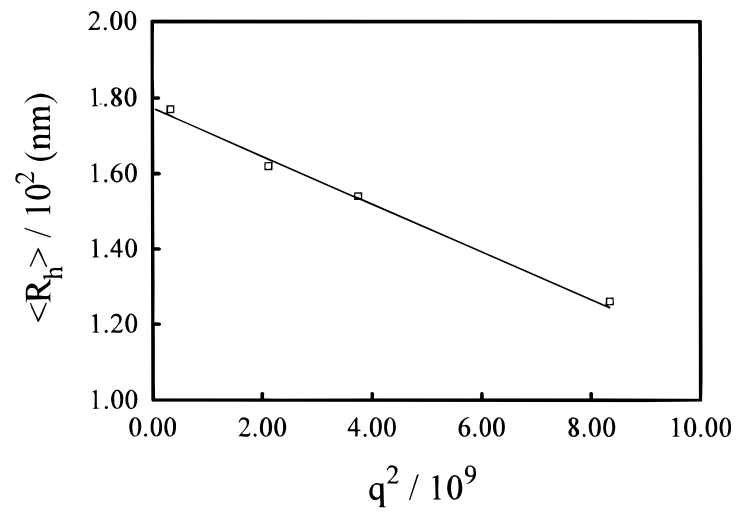

Figure 3. Angular dependence of the average hydrodynamic radius $\left\langle R_{h}\right\rangle$ of the nanofibers in THF, where the nanofiber concentration is $6 \times 10^{-6} \mathrm{~g} / \mathrm{mL}$.

5). It can be seen that the corrections in this case are insignificant.

At a very small angle, i.e., $\mathrm{qR}_{\mathrm{g}} \ll 1$, the translational diffusion is decoupled from both rotational diffusion and internal motions of the nanofibers in dynamic LLS. In this way, the line width $\Gamma$ is only related to the translational diffusion coefficient $\mathrm{D}$ by $\Gamma=\mathrm{Dq}^{2}$. Figure 2 shows a typical translational diffusion coefficient distribution of the nanofi bers in toluene at $6^{\circ}$. Further, $D$ can be converted to the hydrodynamic radius $R_{h}$ by the Stokes-Einstein equation: $R_{h}=k_{B} T / 6 \pi \eta D$ where $\mathrm{k}_{\mathrm{B}}, \eta$, and $\mathrm{T}$ are the Boltzman content, the solvent viscosity, and the absol ute temperature, respectively. ${ }^{10}$ Figure 3 shows that at $\theta=6^{\circ}$ (the left-most point), $\left\langle R_{h}\right\rangle$ is essentially the same as $\left\langle R_{h}\right\rangle_{q \rightarrow 0}$. In infinite dilution, $\left\langle R_{h}\right\rangle=178 \mathrm{~nm}$. It should be noted that such obtained $\left\langle R_{h}\right\rangle$ is an apparent one since the shape of the nanofibers are not sphereic. The ratio of $\left\langle R_{g}\right\rangle /\left\langle R_{h}\right\rangle \sim 1.22$ indicates that the nanofibers do not have an extended thin-rod conformation, which is different from what we expected.

Next, let us estimate the composition of the nanofibers on the basis of our LLS results. Considering the nanofibers as a rigid rod, we estimated its length ( $L$ ) to be $\sim 750 \mathrm{~nm}$ from its radius of gyration, i.e., from $\left\langle\mathrm{R}_{\mathrm{g}}{ }^{2}\right\rangle$ $\sim L^{2} / 12$. On the other hand, we know that for a rigid

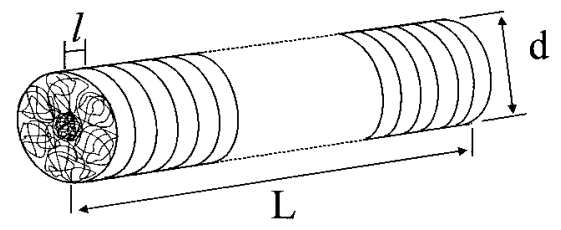

Figure 4. Schematic composition of the nanofiber in toluene.

rod with a diameter of $d$ and a length of $L, D=\left(k_{0} T /\right.$ $3 \eta \mathrm{L}) \ln (\mathrm{L} / \mathrm{d})$, i.e., $\mathrm{L}=2 \mathrm{R}_{\mathrm{h}} \ln (\mathrm{L} / \mathrm{d})$, so that $\mathrm{L} / \mathrm{d} \sim 8$ or $\mathrm{d}$ $\sim 91 \mathrm{~nm}$. Therefore, the nanofibers have a thick rod conformation in solution, which al so explains why $\left\langle\mathrm{R}_{\mathrm{g}}\right\rangle /$ $\left\langle R_{h}\right\rangle$ is very low. Transmission electron microscopy graph showed that the cylindrical phase is hexagonal and the nanofiber has a diameter of $\sim 25 \mathrm{~nm}$ in its dried state. Further, considering that, in the hexagonal cylindrical phase, ${ }^{4}$ each layer consists of six copolymer chains, as shown in Figure 4, we estimated that each nanofiber, on average, is made of $\sim 36$ layers and each layer has a thickness (I) of $\sim 21 \mathrm{~nm}$ because each fiber contains $\sim 220$ copolymer chains. The values of $d$ and I reasonably agree with those estimated from the polystyrene chains with a similar molar mass in toluene. The fact that $\mathrm{d} / \mathrm{l} \sim 4.5$ indicates that the PS blocks are highly stretched in toluene, which is expected because toluene is a good solvent for polystyrene. Further, the average density of the nanofibers estimated from its average mass and hydrodynamic radius is $\sim 2.8 \times 10^{-2}$ $\mathrm{g} / \mathrm{cm}^{3}$ which is slightly higher than that for the polystyrene chains free in toluene because here only the PS blocks can swell, but not the cross-linked PCEMA blocks.

Acknowledgment. The financial support of this work by the RGC (Research Grants Council of Hong Kong Government) Research Assistantship (CUHK 4181/ 97P), a CUHK postdoctoral fellowship for M.L., and a National Distinguished Young Investigator Grant are gratefully acknowledged.

\section{References and Notes}

(1) Block Copolymers, Overview and Critical Survey; Noshay, A., Mcgrath, J . E., Eds.; Academic Press: New York, 1977; p 56.

(2) Brown, R.; Masters, A.; Price, C.; Yuan, X. In Comprehensive Polymer Science; Allen, G., Bevington, J ., E ds., Pergamon: Oxford, England, 1989; Vol. 2, p 155.

(3) Gast, A. P. In Scientific Methods for the Study of Polymer Colloids and Their Applications; Candau, F., Ottewill, R. H., Eds.; Kluwer Academic Publishers: Amsterdam, 1990.

(4) Liu, G.; Qiao, L.; Guo, A. Macromolecules 1996, 29, 5508.

(5) Tao, J .; Guo, A.; Liu, G. Macromolecules 1996, 29, 1618.

(6) Pecora, R.; Berne, J. Dynamic light Scattering; Plenum Press: New York, 1976.

(7) Chu, B. Laser Light Scattering, 2nd ed.; Academic Press: New York 1991.

(8) Zimm, B. H. J . Chem. Phys. 1948, 16, 1099.

(9) Nagai, K. Polym. J . 1972, 3, 67.

(10) Russo, P. S. In Dynamic Light Scattering; Brown, Wyn, Ed.; Oxford University Press: New York, 1993.

(11) Burchard, W.; Schmidt, M.; Stockmayer, W. H. Macromolecules 1980, 13, 1265-1272.

MA9800663 